\title{
Perceptions of Harmfulness of Heated Tobacco Products Compared to Combustible Cigarettes among Adult Smokers in Japan: Findings from the 2018 ITC Japan Survey
}

\author{
Shannon Gravely ${ }^{1, *}$, Geoffrey T. Fong ${ }^{1,2} \mathbb{D}^{\mathbb{D}}$, Edward Sutanto ${ }^{3} \mathbb{(}$, Ruth Loewen ${ }^{1}$, Janine Ouimet ${ }^{1}$, \\ Steve S. Xu ${ }^{1}$, Anne C. K. Quah ${ }^{1}$, Mary E. Thompson ${ }^{4}$, Christian Boudreau ${ }^{4}$, Grace Li ${ }^{1}$, \\ Maciej L. Goniewicz ${ }^{3}$ (D) Itsuro Yoshimi ${ }^{5}$, Yumiko Mochizuki ${ }^{6}$, Tara Elton-Marshall ${ }^{7,8,9,10}$, \\ James F. Thrasher ${ }^{11,12}$ and Takahiro Tabuchi ${ }^{13}$ \\ 1 Department of Psychology, University of Waterloo, Waterloo, ON N2L 3G1, Canada; \\ gfong@uwaterloo.ca (G.T.F.); rloewen@uwaterloo.ca (R.L.); j2ouimet@uwaterloo.ca (J.O.); \\ s4xu@uwaterloo.ca (S.S.X.); ackquah@uwaterloo.ca (A.C.K.Q.); y47li@uwaterloo.ca (G.L.) \\ 2 Ontario Institute for Cancer Research, Toronto, ON M5G 0A3, Canada \\ 3 Division of Cancer Prevention and Population Sciences, Department of Health Behaviors, \\ Roswell Park Comprehensive Cancer Center, Buffalo, NY 14263, USA; \\ edward.sutanto@roswellpark.org (E.S.); maciej.goniewicz@roswellpark.org (M.L.G.) \\ 4 Department of Statistics and Actuarial Science, University of Waterloo, Waterloo, ON N2L 3G1, Canada; \\ methompson@uwaterloo.ca (M.E.T.); cboudreau@uwaterloo.ca (C.B.) \\ 5 Division of Tobacco Policy Research, National Cancer Center Japan, 5-1-1 Tsukiji, Chuo-ku, Tokyo 104-0045, \\ Japan; iyoshimi@ncc.go.jp \\ 6 Japan Cancer Society, 13th Floor, Yurakucho Center Bldg. 2-5-1, Yurakucho, Chiyoda-ku, Tokyo 100-0006, \\ Japan; mochizuki@jcancer.jp \\ 7 Institute for Mental Health Policy Research, Centre for Addiction and Mental Health, London, ON N6G 4X8, \\ Canada; Tara.EltonMarshall@camh.ca \\ 8 Dalla Lana School of Public Health, University of Toronto, Toronto, ON M5T 3M7, Canada \\ 9 Department of Epidemiology and Biostatistics, Schulich School of Medicine and Dentistry, \\ Western University, London, ON N6A 5C1, Canada \\ 10 Ontario Tobacco Research Unit, Toronto, ON M5S 2S1, Canada \\ 11 Department of Health Promotion, Education and Behavior, Arnold School of Public Health, \\ University of South Carolina, Columbia, SC 29208, USA; THRASHER@mailbox.sc.edu \\ 12 Tobacco Research Department, Center for Population Health Research, National Institute of Public Health, \\ Cuernavaca, Morelos 62100, Mexico \\ 13 Cancer Control Center, Osaka International Cancer Institute, Chome-1-69 Otemae, Chuo Ward, \\ Osaka 541-8567, Japan; tabuchitak@gmail.com \\ * Correspondence: shannon.gravely@uwaterloo.ca; Tel.: +15-19-888-4567
}

Received: 9 March 2020; Accepted: 29 March 2020; Published: 1 April 2020

Abstract: In Japan, the tobacco industry promotes heated tobacco products (HTPs) as a reduced-risk tobacco product. This study examines: (1) smokers' harm perceptions of HTPs relative to combustible cigarettes; (2) differences in relative harm perceptions between exclusive smokers and smokers who use HTPs (concurrent users) and between concurrent users based on frequency of product use; and (3) if smokers who were exposed to HTP advertising hold beliefs that are consistent with marketing messages of lower harmfulness. This cross-sectional study included 2614 adult exclusive cigarette smokers and 986 concurrent users who reported their perceptions of harmfulness of HTPs compared to cigarettes, as well as their exposure to HTP advertising in the last six months. Among all smokers, $47.5 \%$ perceive that HTPs are less harmful than cigarettes, $24.6 \%$ perceive HTPs to be equally as harmful, $1.8 \%$ perceive HTPs as more harmful, and $26.1 \%$ did not know. Concurrent users are more likely than exclusive smokers to believe that HTPs are less harmful $(62.1 \%$ versus $43.8 \%, p<0.0001)$ 
and less likely to report that they did not know $(14.3 \%$ versus $29.4 \%, p<0.0001)$. Frequent HTP users are more likely than infrequent users to believe that HTPs are less harmful $(71.7 \%$ versus $57.1 \%, p \leq 0.001)$. Believing that HTPs are less harmful than cigarettes was associated with noticing HTP advertising on TV $(p=0.0005)$, in newspapers/magazines $(p=0.0001)$, on posters/billboards $(p<0.0001)$, in stores where tobacco $(p<0.0001)$ or where HTPs $(p<0.0001)$ are sold, on social media $(p<0.0001)$, or in bars/pubs $(p=0.04)$. HTP users were significantly more likely than non-HTP users to believe that HTPs are less harmful than cigarettes, with this belief being more prominent among frequent users. Smokers who have been exposed to HTP advertising were more likely to perceive HTPs as less harmful than cigarettes.

Keywords: heated tobacco products; heat-not-burn; modified risk tobacco products; combustible cigarettes; perceptions of harm; risk

\section{Introduction}

Smoked tobacco is the most dangerous and common form of nicotine consumption [1,2]. The smoke from cigarettes includes over 4000 chemicals and at least 70 known carcinogens [3]. Tobacco harm reduction is a public health strategy that aims to lower exposure to the toxicants produced by tobacco smoke, by encouraging smokers to completely substitute cigarettes with a less harmful product that can deliver nicotine. Alternative nicotine products, such as e-cigarettes and snus, can effectively deliver nicotine without combustion, and are considered to be less harmful than cigarettes [4-7]. Notably, the complete substitution of reduced-risk alternatives for cigarettes may offer a substantial reduction in smoking prevalence and mortality if widely adopted $[8,9]$.

Heated tobacco products (HTPs) are an example of an alternative tobacco product that has been developed by the tobacco industry and may be potentially less harmful than cigarettes $[6,7,10-13]$. HTPs heat tobacco in loose leaf form or contained in tobacco sticks, plugs, or capsules using a battery-powered heating system. Tobacco is heated (not combusted like traditional cigarettes) to generate an inhalable nicotine-containing aerosol [12,14].

HTPs are not a new concept. They were first introduced in the 1980s, but unsuccessfully [12]. A newer generation of HTPs (e.g., IQOS from Philip Morris International (PMI)) has been introduced in many countries around the world. HTP market growth has generally been slow in most countries, with Japan being an exception [15]. HTPs were introduced into Japan's open market in 2016, initially with IQOS, which was followed by Ploom TECH from Japan Tobacco and glo from British American Tobacco in 2017. According to a market report by PMI, IQOS had captured $17.4 \%$ of Japan's tobacco share in 2019 [16], and although Japan's HTP market has expanded with other HTPs, IQOS still controls about $70 \%$ of the HTP market in Japan [16]. The growing popularity of HTPs in Japan (accounting for 24.3\% of Japan's tobacco market in March 2019 [16]) may in part be due to the prohibition of nicotine e-cigarettes; thus, there is no competition between HTPs and e-cigarettes.

The tobacco industry has been actively marketing HTPs in Japan as a reduced-risk tobacco product, due to its purported non-combustible nature [11]. However, there is currently no scientific consensus that HTPs are safer than cigarettes [12-14], or if they will be a successful smoking cessation aid [14]. Some comprehensive reviews of the literature have suggested that HTPs may be less harmful than cigarettes because they are likely to expose users and bystanders to lower levels of particulate matter and harmful compounds [7,12,13,17], but the World Health Organization (WHO) strongly debates that they should be used as an alternative to cigarettes based on the reduced-risk assertion made by the tobacco industry [18]. Despite the current scientific uncertainty about HTPs' absolute or relative harmfulness compared to cigarettes (mainly owing to large variability of reduced toxicant profiles between studies) [7], Japanese law does not prevent them from being marketed as a reduced-risk product. 
It is currently unclear how smokers in Japan perceive the harmfulness of HTPs relative to cigarettes and how exposure to marketing may be associated with harmfulness perceptions. Thus, the aims of this study were to examine: (1) smokers' harm perceptions of HTPs relative to cigarettes; (2) differences in relative harm perceptions between: (i) exclusive smokers and smokers who use HTPs (concurrent users); (ii) concurrent users based on HTP use frequency (frequent versus infrequent HTP users); and (iii) concurrent users based on HTP and smoking frequency; and (3) whether smokers' beliefs about lower harmfulness are associated with exposure to HTP advertising.

\section{Methods}

\subsection{Sample, Study Design, and Procedure}

The International Tobacco Control (ITC) Wave 1 Japan Survey (conducted February-March 2018) was a web-administered survey of behaviors and attitudes related to tobacco and nicotine use among a nationally representative sample of 4615 adult (aged 20+) Japanese exclusive cigarette smokers (smoke at least monthly), exclusive HTP users (use HTPs at least weekly), concurrent HTP users and cigarette smokers, and non-users (do not smoke or use HTPs). The survey was conducted with Rakuten Insight's proprietary online panel in Japan, with quotas for region of residence, gender, and age, to ensure that the final sample was proportional to stratum sizes from Japan census data. Recruitment for the panel was conducted on a daily basis, tapping into users of Rakuten services (e.g., e-commerce, credit cards, insurance, mobile services, etc.), as well as other online resources such as affiliates, email and banner recruits in order to maintain a panel as consistent as possible with the general population. Panelists received email invitations and also had the option of logging into their proprietary panel site to access the survey.

The overall survey response rate was $45.1 \%$ (with a $96.3 \%$ cooperation rate). The survey protocols and all materials, including the survey questionnaires, were cleared for ethics by Office of Research Ethics, University of Waterloo, Canada (ORE\#22508/31428). All participants provided consent to participate. Further methodological details can be found in the Wave 1 ITC Japan technical report (Japan Wave 1 Technical Report).

For the present cross-sectional analyses, respondents were included if, at the time of the survey they: (1) had ever heard of HTPs; (2) were current cigarette smokers (smoked at least monthly); and (3) had complete outcome data for the questions about harmfulness of HTPs compared to cigarettes, and exposure to HTP marketing. Of the overall sample of 4615 Wave 1 respondents, 3838 met the inclusion criteria of being a current (at-least monthly) smoker. Of these, 3600 respondents had heard of HTPs and completed the relevant outcome questions; 2614 were exclusive cigarette smokers; and 986 were concurrent users (see the Study Flow Diagram: Figure S1).

\subsection{Measures}

The survey, with original response options, can be found at the ITC Project website: ITC JP 1 Survey. The following variables were used in the current study:

\subsubsection{Sociodemographic Variables}

Sociodemographic measures were: gender (female versus male), age group (20-29, 30-39, 40-59, $\geq 60$ years), income [low: 4,000,000 yen or less; medium: more than 4,000,000 yen to 6,000,000 yen; high: more than 6,000,000 yen; or not reported], and education [low: junior high school, high school; medium: vocational school, junior college/ technical college; high: undergraduate or higher; or not reported].

\subsubsection{Smoking Variables}

Respondents reported their cigarette smoking frequency at the time of the survey and were categorized as a daily or non-daily (weekly or monthly) smoker. 


\subsubsection{HTP Use Variables}

Respondents reported whether they had ever heard of HTPs: 'yes' or 'no'; those who reported that they did not know were classified as 'no'. If respondents had heard of HTPs they were asked: "Have you ever used one of these heat-not-burn products, even one time?" ('yes' or 'no'). If respondents reported ever using an HTP, they were asked about frequency of use: "How often do you CURRENTLY use heat-not-burn products? These include products such as IQOS, Ploom TECH, and glo". Response option were: daily, weekly, monthly, less than monthly, or not at all. Current HTP use was classified as: 'any current HTP use' (daily, weekly, monthly, or less than monthly) or 'not currently using an HTP' (tried it/used it in the past, or never tried one).

\subsubsection{Smoker and/or HTP User Groups}

Smokers were divided into two main user groups:

1. Exclusive Smoker: currently smokes cigarettes at least monthly AND does not currently use an HTP.

2. Concurrent User: currently smokes cigarettes at least monthly AND currently uses an HTP (any current HTP use).

\subsubsection{Concurrent User Groups}

Concurrent users were further categorized using two methods, based on frequency of smoking and HTP use:

1. HTP frequency only: 'Frequent HTP user' (at least weekly use) versus 'Infrequent HTP user' (less than weekly use).

2. HTP and smoking frequency, using the classification system derived in Borland et al. (because daily nicotine users differ considerably from non-daily nicotine users) [19]: (i) 'Predominant smoker': Daily smoking and less-than-daily HTP use; (ii) 'Concurrent-daily user': Daily smoking and daily HTP use; (iii) 'Concurrent non-daily user': Less-than-daily smoking and less-than-daily HTP use; or (iv) 'Predominant HTP user': Less than daily smoking and daily HTP use.

\subsubsection{Perceived Harmfulness of HTPs Relative to Cigarettes}

Respondents who were aware of HTPs were asked the following question: "Compared to smoking cigarettes, how harmful do you think using a heat-not-burn tobacco product is?". Responses options included: 'Much less harmful than smoking cigarettes', 'Somewhat less harmful than smoking cigarettes', 'Equally harmful to smoking cigarettes', 'Somewhat more harmful than smoking cigarettes', or 'I do not know'. These responses were re-categorized as: 'HTPs are less harmful than cigarettes', 'HTPs are equally harmful as cigarettes', 'HTPs are more harmful than cigarettes', or 'I do not know'.

\subsubsection{Exposure to HTP Ads Via Various Marketing Platforms}

Respondents answered the question "In the last six months have you noticed heat-not-burn products being advertised in any of the following places?": TV; radio; newspapers/magazines; posters/billboards; stores where tobacco products are sold; stores where HTPs are sold; social media; and bars/pubs. Responses were categorized as: 'yes' versus 'no/ I do not know'.

\subsection{Statistical Analyses}

Unweighted data were used to describe the study sample. Chi-square tests were conducted overall and between the user groups (exclusive smokers versus concurrent users). All subsequent analyses were conducted using weighted data. Cross-sectional weights were computed for all respondents. A raking algorithm was used to calibrate the weights on smoking status, HTP use, geographic region, and demographic measures (e.g., gender, age, ethnicity, and education) [20]. 
In the first set of analyses, two multinomial regression models were conducted to compute weighted and adjusted estimates for perceived relative harmfulness of HTPs compared to cigarettes (HTPs are less harmful versus equally harmful versus more harmful versus I do not know). Model 1 (objective 1) included all respondents and controlled for: gender, age, income, education, smoking frequency (daily versus non-daily) and frequency of HTP use (daily versus non-daily versus not using one). In Model 2 (objective 2 (i)), perceptions of harmfulness were examined by user group status (exclusive smokers versus concurrent users). The model controlled for: gender, age, income, education, and smoking frequency (daily versus non-daily). A sub-analysis was conducted using adjusted weighted logistic regression models to estimate the odds of believing that HTPs are less harmful than cigarettes (compared to equally harmful/more harmful/I do not know) for all respondents, as well as broken down by user group: exclusive smokers and concurrent users.

The second set of analyses was restricted to the concurrent user group, those who both smoke and use HTPs. The first multinomial regression (objective 2 (ii)) compared perceptions of relative harmfulness between frequent HTP users (at least weekly use) and infrequent HTP users (less than weekly use). The outcome was re-categorized as: less harmful versus more/equally harmful versus I do not know, in order to account for low proportion of respondents who reported that HTPs were more harmful than cigarettes (2.4\%). The model controlled for gender, age, income, education, and smoking frequency (daily versus non-daily). The second multinomial regression model (objective 2 (iii)) examined difference between concurrent users based on smoking and HTP use frequency and compared: predominant smokers versus concurrent-daily users versus concurrent non-daily users. Due to the small sample size of concurrent non-daily users $(n=58)$, we used a dichotomized outcome (HTPs are less harmful versus HTPs are equally/more harmful/I do not know). Predominant HTP users were excluded due to the small sample size $(n=4)$. The model controlled for gender, age, income, and education.

The final set of analyses (objective 3) included all respondents and compared perceptions of relative harm of HTPs (HTPs are less harmful than cigarettes versus equally/more/I do not know) for those who had or had not noticed HTP advertising via various marketing platforms (yes versus no/I do not know). The weighted logistic regression models controlled for gender, age, income, education, and user group (exclusive smokers versus concurrent users).

Statistical significance and confidence intervals were tested at the $95 \%$ confidence level. Analyses were conducted using SAS Version 9.4 (SAS Institute Inc. 2013, Cary, North Carolina, USA).

\section{Results}

Table 1 presents the (unweighted) respondents' characteristics (overall and by user group status). Overall, $76.4 \%$ of the total sample were male, $94.4 \%$ were daily cigarette smokers, and $11.1 \%$ were current daily HTP users. The majority of the sample was aged $40+(67.2 \%)$, with concurrent users being significantly younger than exclusive smokers $(p<0.0001)$. Among the exclusive smokers $(n=2614)$, $94.7 \%$ smoked daily; among concurrent users $(n=986), 93.7 \%$ smoked daily, and $40.6 \%$ used HTPs daily. 
Table 1. Unweighted sample characteristics of respondents at Wave 1 (2018).

\begin{tabular}{|c|c|c|c|c|c|c|}
\hline \multicolumn{2}{|c|}{ Chacteristics } & \multirow{2}{*}{$\begin{array}{c}\text { Exclusive Smokers } \\
n=2614(72.6) \\
n(\%) \\
2026(77.5) \\
588(22.5) \\
\end{array}$} & \multirow{2}{*}{$\begin{array}{c}\text { Concurrent Users } \\
n=986(27.4) \\
n(\%) \\
723(73.3) \\
263(26.7)\end{array}$} & \multirow{2}{*}{$\begin{array}{c}\begin{array}{c}\text { Chi-Square }\left(\chi^{2}\right) \text { Test } \\
\text { between User Groups }\end{array} \\
\chi^{2}=6.9, p=0.009\end{array}$} & \multirow{2}{*}{$\begin{array}{c}\text { All Respondents } \\
n=3600 \\
n(\%) \\
2749(76.4) \\
851(23.6)\end{array}$} & \multirow{2}{*}{$\begin{array}{c}\begin{array}{c}\text { Overall } \\
\text { Chi-Square }\end{array} \\
p<0.0001\end{array}$} \\
\hline Gender & $\begin{array}{c}\text { Male } \\
\text { Female }\end{array}$ & & & & & \\
\hline Age group (years) & $\begin{array}{l}20-29 \\
30-39 \\
40-59 \\
\geq 60\end{array}$ & $\begin{array}{c}197(7.5) \\
494(18.9) \\
1198(45.8) \\
725(27.7)\end{array}$ & $\begin{array}{l}178(18.1) \\
310(31.4) \\
372(37.7) \\
126(12.8)\end{array}$ & $\chi^{2}=205.0, p<0.0001$ & $\begin{array}{c}375(10.4) \\
804(22.3) \\
1570(43.6) \\
851(23.6)\end{array}$ & $p<0.0001$ \\
\hline Education level & $\begin{array}{c}\text { Low } \\
\text { Medium } \\
\text { High } \\
\text { Not reported }\end{array}$ & $\begin{array}{c}993(38.0) \\
435(16.6) \\
1158(44.3) \\
28(1.1)\end{array}$ & $\begin{array}{c}318(32.3) \\
168(17.0) \\
495(50.2) \\
5(0.5)\end{array}$ & $\chi^{2}=52.4, p<0.0001$ & $\begin{array}{c}1311(36.4) \\
603(16.8) \\
1653(45.9) \\
33(0.9)\end{array}$ & $p<0.0001$ \\
\hline Income & $\begin{array}{c}\text { Low } \\
\text { Medium } \\
\text { High } \\
\text { Not reported } \\
\end{array}$ & $\begin{array}{l}727(27.8) \\
571(21.8) \\
956(36.6) \\
360(13.8)\end{array}$ & $\begin{array}{c}189(19.2) \\
247(25.1) \\
457(46.4) \\
93(9.4)\end{array}$ & $\chi^{2}=14.5, p=0.002$ & $\begin{array}{c}916(25.4) \\
818(22.7) \\
1413(39.3) \\
453(12.6)\end{array}$ & $p<0.0001$ \\
\hline Smoking frequency & $\begin{array}{c}\text { Daily } \\
\text { Non-daily }\end{array}$ & $\begin{array}{c}2476(94.7) \\
5.3(5.3)\end{array}$ & $\begin{array}{c}924(93.7) \\
6.3(6.3) \\
\end{array}$ & $\chi^{2}=1.4, p=0.24$ & $\begin{array}{c}3400(94.4) \\
200(5.6)\end{array}$ & $p<0.0001$ \\
\hline HTP use frequency & $\begin{array}{c}\text { Daily } \\
\text { Weekly } \\
\text { Monthly } \\
\text { Less than monthly } \\
\text { Not at all }\end{array}$ & $\begin{array}{c}- \\
- \\
- \\
2614(100.0)\end{array}$ & $\begin{array}{c}400(40.6) \\
148(15.0) \\
94(9.5) \\
344(34.9) \\
-\end{array}$ & $\mathrm{N} / \mathrm{A}$ & $\begin{array}{c}400(11.1) \\
148(4.1) \\
94(2.6) \\
344(9.6) \\
2614(72.6)\end{array}$ & $p<0.0001$ \\
\hline Concurrent User Group & $\begin{array}{l}\text { Predominant smoker } \\
\text { Concurrent-daily user } \\
\text { Concurrent non-daily user } \\
\text { Predominant HTP user }\end{array}$ & $\begin{array}{l}- \\
- \\
-\end{array}$ & $\begin{array}{c}396(40.2) \\
528(53.5) \\
58(5.9) \\
4(0.4)\end{array}$ & $\mathrm{N} / \mathrm{A}$ & $\begin{array}{c}528(14.7) \\
396(11.0) \\
58(1.6) \\
4(0.1)\end{array}$ & $p<0.0001$ \\
\hline
\end{tabular}

Predominant smokers: Smoke cigarettes daily and use HTPs less than daily; Concurrent-daily users: Smoke cigarettes and use an HTP daily; Concurrent non-daily users: does not smoke cigarettes or use an HTP daily; predominant HTP user: daily HTP use; non-daily smoker. HTP: Heated tobacco product. N/A: Not applicable. 


\subsection{Smokers' Perceptions of Harmfulness of HTPs Relative to Cigarettes}

Of the 3600 smokers, $47.5 \%$ perceived that HTPs were less harmful than cigarettes, $24.6 \%$ perceived HTPs to be equally as harmful than cigarettes, $1.8 \%$ perceived HTPs as more harmful, and $26.1 \%$ did not know (Figure 1 and Table S1).

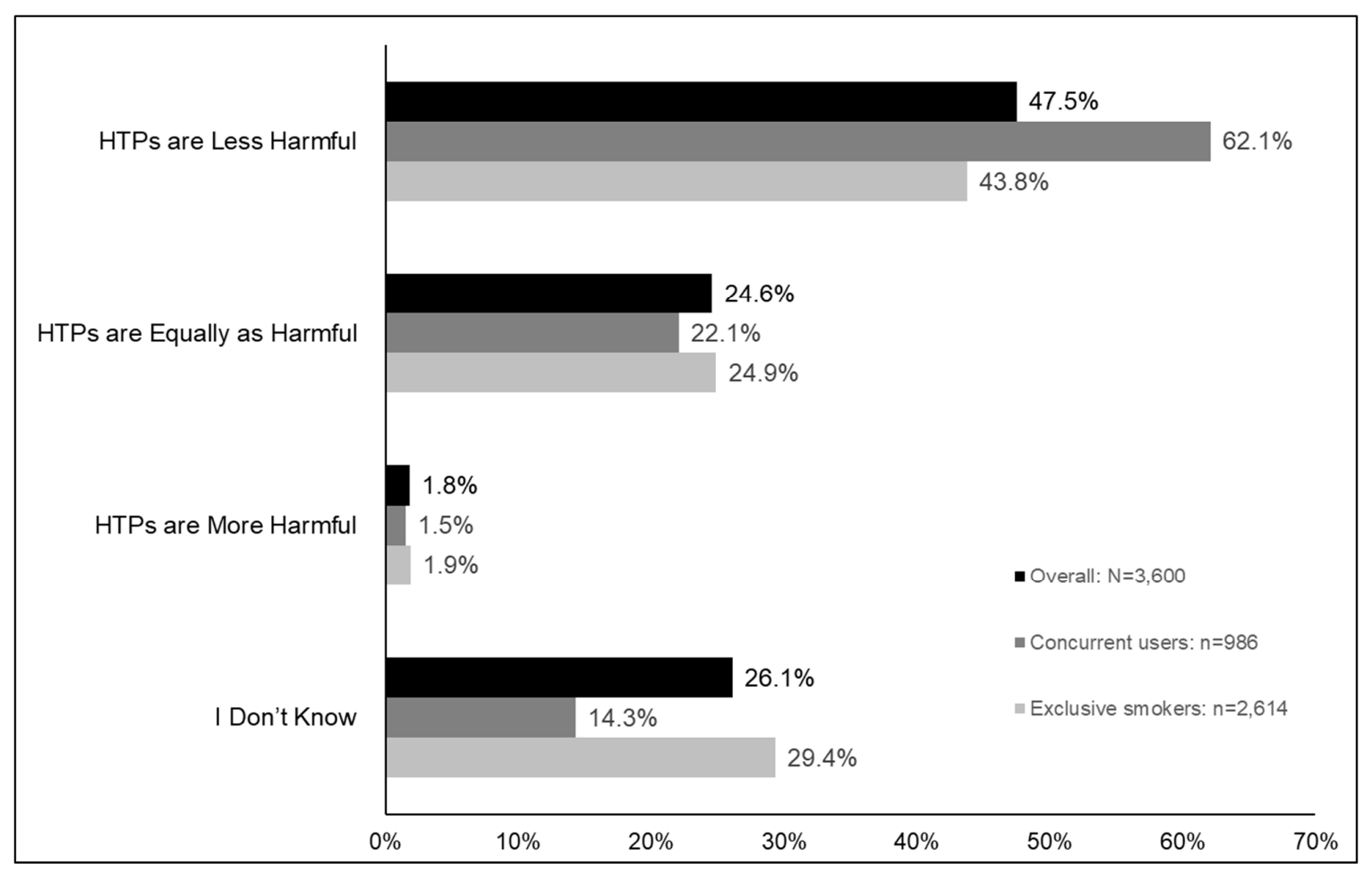

Figure 1. Relative perceptions of harmfulness of HTPs compared to cigarettes among smokers in Japan in 2018.

Differences in Relative Harm Perceptions between Exclusive Smokers and Concurrent Users

When user groups were compared, concurrent users were significantly more likely than exclusive smokers to believe that HTPs are less harmful $(62.1 \%$ versus $43.8 \%, p<0.0001)$ and significantly less likely than exclusive smokers to report that they did not know ( $14.3 \%$ versus $29.4 \%, p<0.0001)$ (Figure 1 and Table S1).

\subsection{Odds of Believing That HTPs Are Less Harmful than Cigarettes: Exclusive Smokers versus Concurrent Users}

Concurrent users were twice as likely as exclusive smokers to believe that HTPs are less harmful, compared to being equally harmful, more harmful, or uncertain (odds ratio $(\mathrm{OR})=2.1,95 \%$ confidence interval (CI): 1.7-2.5), which is likely being driven by the more frequent HTP users, who are more likely to perceive that HTPs are less harmful than infrequent HTP users (OR $=1.9,95 \%$ CI: 1.4-2.6). Concurrent daily users were twice as likely as predominant smokers (OR $=0.5,95 \%$ CI: $0.4-0.7$ ) and as concurrent non-daily users (OR $=0.5,95 \%$ CI: $0.2-0.9)$ to believe that HTPs are less harmful (Table 2). 
Table 2. Adjusted (Weighted) regression models estimating the odds of believing that HTPs are less harmful than cigarettes among smokers in Japan in 2018.

\begin{tabular}{|c|c|c|c|c|}
\hline \multirow{2}{*}{$\begin{array}{c}\text { User Group } \\
\text { All Users }(N=3600)\end{array}$} & \multirow{2}{*}{$\begin{array}{l}\text { HTPs Are Less } \\
\text { Harmful \% }\end{array}$} & \multirow{2}{*}{ Odds Ratio } & \multicolumn{2}{|c|}{$95 \% \mathrm{CI}$} \\
\hline & & & Lower CI & Upper CI \\
\hline Exclusive Smokers $(n=2614)$ & $43.8 \%$ & Reference & & \\
\hline Concurrent Users $(n=986)$ & $62.1 \%$ & 2.1 & 1.7 & 2.5 \\
\hline Concurrent Users $(n=986)$ & & & & \\
\hline Frequent HTP Users $(n=548)$ & $71.7 \%$ & 1.9 & 1.4 & 2.6 \\
\hline Infrequent HTP Users (438) & $57.1 \%$ & Reference & & \\
\hline Concurrent Users $(n=982)$ & & & & \\
\hline Predominant Smokers $(n=528)$ & $58.8 \%$ & 0.5 & 0.4 & 0.7 \\
\hline Concurrent Daily Users $(n=396)$ & $73.2 \%$ & Reference & & \\
\hline Concurrent Non-daily Users $(n=58)$ & $56.4 \%$ & 0.5 & 0.2 & 0.9 \\
\hline
\end{tabular}

Outcome: Less harmful versus equally/more harmful/do not know. HTPs: Heated tobacco products; CI: Confidence interval.

\subsubsection{Differences in Relative Harm Perceptions Between Concurrent Users}

Differences in relative harm perceptions by HTP use frequency: When concurrent users were categorized by frequency of HTP use, frequent users were significantly more likely than infrequent users to believe that HTPs are less harmful than cigarettes $(71.7 \%$ versus $57.1 \%, p<0.001)$. More of the infrequent HTP users reported that they did not know, compared to frequent users $(13.6 \%$ versus $9.0 \%$, $p<0.01$ ) (Figure 2 and Table S2).

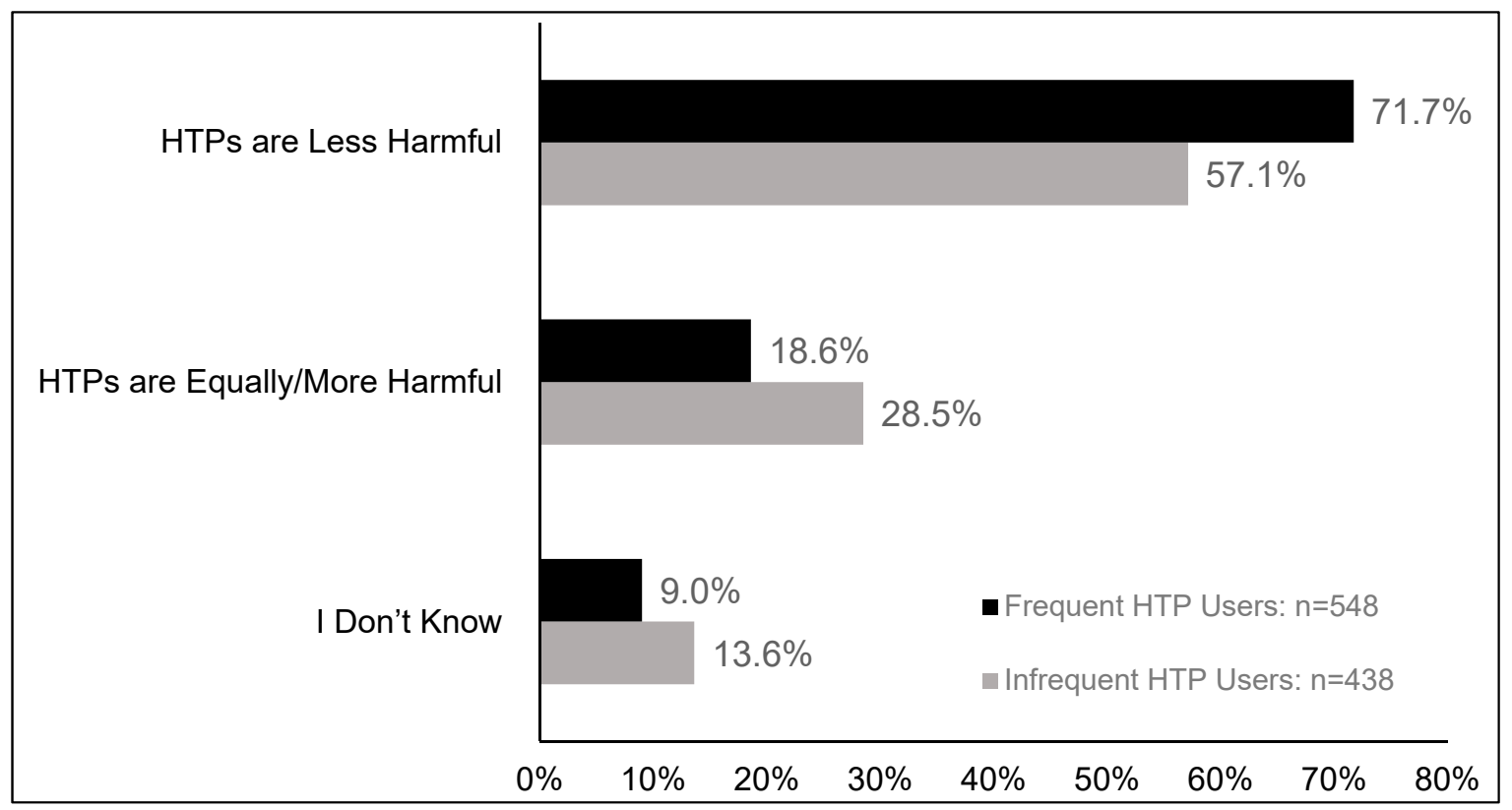

Figure 2. Relative perceptions of harmfulness of HTPs compared to cigarettes among concurrent users stratified by HTP use frequency (frequent HTP users versus infrequent HTP users). Data are weighted and adjusted. HTPs: Heated tobacco products. Frequent HTP user: at least weekly use; infrequent HTP user: less than weekly use.

Differences in relative harm perceptions by frequency of HTP use and smoking: When concurrent users were compared by the frequency of HTP use and smoking, the analysis showed there was a main effect between these groups ( $p=0.0005)$. Concurrent daily users were more likely to believe HTPs are less harmful than both predominant smokers $(73.2 \%$ versus $58.8 \%, p<0.001)$ and concurrent non-daily users $(56.4 \%, p=0.03)$ (Figure 3 and Table S3). 


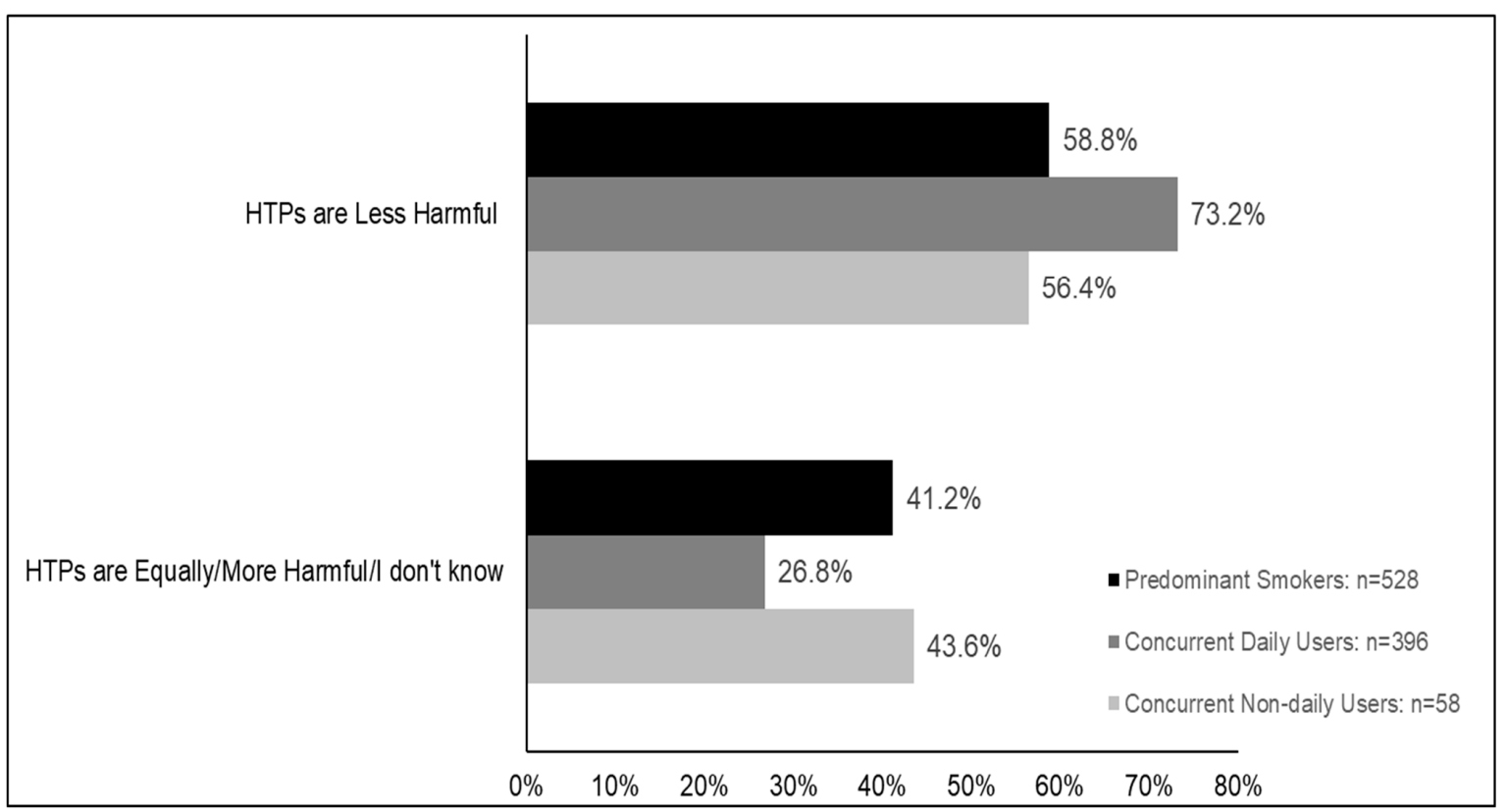

Figure 3. Relative perceptions of harmfulness of HTPs compared to cigarettes among concurrent users stratified by smoking and HTP use frequency (Predominant Smokers vs. Concurrent-daily Users vs. Concurrent Non-daily Users). Data are weighted and adjusted. HTPs: Heated tobacco products. Predominant Smokers: smoke cigarettes daily and use HTP less than daily; Concurrent-daily users: smoke cigarettes and use HTP daily; Concurrent non-daily users: smoke cigarettes and use HTP less than daily.

\subsubsection{Exposure to Various Marketing Platforms that Advertise HTPs}

Smokers who reported noticing (e.g., were exposed to) HTP marketing in the last six months, compared to those who did not notice, were more likely to believe that HTPs are less harmful, for nearly all venues where marketing was observed: TV (OR $=1.4,95 \%$ CI: $1.1-1.6, p<0.001)$, newspapers or magazines $(\mathrm{OR}=1.4,95 \% \mathrm{CI}: 1.2-1.7, p=0.0001)$, posters or billboards $(\mathrm{OR}=1.4,95 \% \mathrm{CI}: 1.2-1.7$, $p<0.0001)$, stores where tobacco (OR $=1.7,95 \% \mathrm{CI}: 1.4-2.0, p<0.0001)$ or HTPs $(\mathrm{OR}=1.5,95 \%$ CI: $1.3-1.8, p<0.0001)$ are sold, social media (OR = 1.6, 95\% CI: $1.3-1.9, p<0.0001$ ), and bars/pubs $(\mathrm{OR}=1.3,95 \% \mathrm{CI}: 1.0-1.7, p=0.045)$. There were no differences between those who noticed HTP advertising on the radio and those who did not $(p=0.35)$ (Table 3). 
Table 3. Adjusted (weighted) regression models estimating the odds of believing that HTPs are less harmful than cigarettes among smokers in Japan in 2018 based on exposure to various marketing platforms $(n=3600)$.

\begin{tabular}{|c|c|c|c|c|c|c|}
\hline \multirow{2}{*}{ Advertising Location } & \multirow{2}{*}{ Exposure } & \multirow{2}{*}{$\begin{array}{c}\text { HTPs Are Less } \\
\text { Harmful \% }\end{array}$} & \multirow{2}{*}{$p$-Value } & \multirow{2}{*}{ Odds Ratio } & \multicolumn{2}{|c|}{$95 \%$ CI } \\
\hline & & & & & Lower CI & Upper CI \\
\hline \multirow[t]{2}{*}{ TV } & Yes $(n=890)$ & $52.1 \%$ & 0.0005 & 1.4 & 1.1 & 1.6 \\
\hline & No $(n=2710)$ & $44.3 \%$ & & Reference & & \\
\hline \multirow[t]{2}{*}{ Radio } & Yes $(n=170)$ & $50.5 \%$ & 0.35 & 1.2 & 0.8 & 1.7 \\
\hline & No $(n=3430)$ & $46.0 \%$ & & Reference & & \\
\hline Newspapers or magazines & Yes $(n=1012)$ & $52.3 \%$ & 0.0001 & 1.4 & 1.2 & 1.7 \\
\hline \multirow[t]{2}{*}{ Posters or billboards } & Yes $(n=1366)$ & $51.9 \%$ & $<0.0001$ & 1.4 & 1.2 & 1.7 \\
\hline & No $(n=2234)$ & $42.9 \%$ & & Reference & & \\
\hline \multirow[t]{2}{*}{ Stores where tobacco is sold } & Yes $(n=2394)$ & $50.6 \%$ & $<0.0001$ & 1.7 & 1.4 & 2.0 \\
\hline & No $(n=1206)$ & $38.3 \%$ & & Reference & & \\
\hline Stores where HTPs are sold & Yes $(n=2164)$ & $50.5 \%$ & $<0.0001$ & 1.5 & 1.3 & 1.8 \\
\hline Bars or pubs & No $(n=3233)$ & $45.6 \%$ & & Reference & & \\
\hline
\end{tabular}

Outcome: Less harmful versus equally/more harmful/do not know (Reference). 


\section{Discussion}

The current study examined perceptions of harmfulness of HTPs compared to cigarettes among a sample of adult Japanese exclusive smokers and concurrent users of cigarettes and HTPs, finding that about half of smokers perceived HTPs as being less harmful than cigarettes. A quarter believed that they are equally as harmful and a quarter did not know. Very few smokers believed that HTPs are more harmful than cigarettes. Concurrent users were twice as likely than exclusive smokers to believe that HTPs are less harmful and were also less likely to report that they did not know. Among concurrent users only, frequent HTP users were twice as likely than infrequent users to believe that HTPs are less harmful, and concurrent daily users were twice as likely as predominant smokers and concurrent non-daily users to believe that HTPs are less harmful than cigarettes. Smokers exposed to HTP advertising via various marketing platforms, including TV, billboards, social media, newspapers, magazines, bars/pubs, and stores where tobacco and HTPs are sold, were more likely to perceive HTPs as less harmful than cigarettes.

Consumer perceptions of HTP harmfulness relative to cigarettes may be a key predictor of initiation and continued use of HTPs. For example, smokers are more likely to switch to or initiate use of other alternative nicotine products (e.g., e-cigarettes) if they believe them to be less harmful than cigarettes [21-23]; however, few studies have addressed this for HTPs. A recent qualitative study of current and former IQOS users in the United Kingdom found that one of the main reasons for initiating HTP use and continued use was because it is 'less harmful', 'less hazardous', and 'less damaging' for their health than cigarettes [24]. A cross-sectional online survey of HTP users from various countries, mainly from Switzerland, reported $89 \%$ of respondents were using HTPs because they are less toxic than cigarettes [25]. Two other research studies in Korea [26] and Germany [27] also reported HTP use was positively associated with believing that they were less harmful than cigarettes. Our results reflect these findings, such that those who reported using HTPs in our study were more likely than non-HTP-users to believe they are less harmful than cigarettes, and this belief was greater among the more frequent HTP users.

Advertising and other promotional approaches are critical to increasing the appeal and use of tobacco products [28]. For example, tobacco image advertising is designed to increase product visibility and to communicate appealing attributes such as attractiveness, performance, identity, and quality [28]. HTPs are no exception, especially in Japan where there are no advertising bans and few product packaging laws.

HTPs are marketed as sophisticated, modern, high tech, and clean reduced-risk products in order to increase appeal, and to minimize health concerns among Japanese consumers (IQOS Ads and Products) $[11,18,29]$. The tobacco industry has used creative marketing strategies via a large number of advertising platforms (e.g., stores, TV, billboards, magazines, and social media) in Japan to attract customers and increase HTP sales [29]. Although our data cannot determine if exposure to various types of HTP advertising impacted HTP relative harm perceptions, or even HTP use, we did find that exposure to HTP advertising was associated with the belief that HTPs are less harmful than cigarettes, and HTP users were more likely to hold this belief compared to non-HTP users.

Our study did not examine whether HTP users are former or never-smokers who started using HTPs because they believe they are less harmful alternative to cigarettes, or if current smokers want to substitute their cigarettes with a potentially less harmful alternative; however, some research has suggested that HTPs may in fact be barrier to smoking cessation in Japan. For example, despite PMI's claims that most IQOS users are former smokers [16], independent research has shown that the majority of Japanese HTP users do not quit smoking [29-32]. For example, Xu et al. found that, among the reasons for using HTPs cited by Japanese concurrent users, non-quit reasons-to cut down on cigarettes $(64.6 \%)$ and to not have to give up cigarettes altogether $(52.1 \%)$ - were mentioned as often as or more often than the desire to quit smoking (55.4\%) [33]. Research has consistently shown that light smoking [34] or reducing cigarette consumption (even among heavy smokers who reduce their daily consumption by $50 \%$ ) does not result in a reduction of smoking-attributable disease and death [35], therefore using HTPs to simply reduce cigarette consumption should be strongly discouraged. 
Although this large survey of Japanese smokers and concurrent users was nationally representative, some limitations exist. First, because this study was cross-sectional, it cannot be used to demonstrate causality. For example, we cannot determine whether perceptions of harm preceded or followed HTP use. Similarly, it is also not possible to determine if smokers changed their beliefs about the relative harm of HTPs compared to cigarettes because they were exposed to HTP marketing, or if they were better able to recall marketing because it was in line with their own beliefs. Longitudinal cohort studies are needed to examine the impact of HTP marketing on harm perceptions, attitudes, and beliefs, and if smokers who perceive HTPs to be less harmful will be more likely to initiate use, and how this may impact subsequent smoking cessation. Secondly, this study only included current smokers, therefore the results from this study cannot be generalizable to former smokers or non-smokers. Third, these results were obtained from adults in Japan, and thus may not apply to other countries or younger age groups.

\section{Conclusions}

Overall, this study found that nearly half of all smokers believed HTPs to be less harmful than cigarettes. HTP users were significantly more likely to believe that HTPs are less harmful than cigarettes compared to non-HTP users, with this belief being more prominent among frequent users. Smokers who have been exposed to HTP marketing appear to hold beliefs that are consistent with these advertising messages in Japan [36]. Even though existing scientific data show less exposure to toxic substances from HTPs than from cigarettes [7,12,13,17], advertising HTPs as reduced-risk products should be prohibited until the scientific community comes to a consensus about the ultimate effect of HTP use on health. Moreover, if the use of HTPs continues to increase in Japan, and possibly in other countries, the provision of balanced information and smoking cessation advice to smokers who are interested in using HTPs in place of combustible cigarettes is imperative. Communication should be framed to differentiate between relative and absolute harms, while providing an evidence-based appraisal of the relative risk of HTPs in comparison to combustible cigarettes.

Supplementary Materials: The following are available online at http://www.mdpi.com/1660-4601/17/7/2394/s1, Figure S1: Study Flow Diagram, Table S1: Relative Perceptions of Harmfulness of HTPs Compared to Cigarettes Among Smokers in Japan in 2018, Table S2: Relative Perceptions of Harmfulness of HTPs Compared to Cigarettes Among Concurrent Users Stratified by HTP Use Frequency (Frequent HTP Users vs. Infrequent HTP Users), Table S3: Relative Perceptions of Harmfulness of HTPs Compared to Cigarettes Among Concurrent Users Stratified by Smoking and HTP Use Frequency: Predominant Smokers Vs. Concurrent Daily Users vs. Concurrent Non-daily Users.

Author Contributions: Conceptualization: S.G.; methodology: M.E.T., C.B., J.O., A.C.K.Q., G.T.F.; formal analysis, S.G.; investigation: I.Y., T.T., Y.M., M.E.T., C.B., G.T.F., A.C.K.Q.; visualization: all authors; data curation: M.E.T., C.B., G.L., A.C.K.Q.; writing-original draft preparation: S.G.; writing-review and editing: all authors; supervision: G.T.F.; project administration: A.C.K.Q., M.E.T., C.B., R.L., G.T.F., I.Y., T.T., Y.M.; funding acquisition: G.T.F., I.Y. All authors have read and agreed to the published version of the manuscript.

Funding: The 2018 ITC Japan Survey was funded by a Canadian Institutes of Health Research Foundation Grant (FDN-148477), with additional funding provided by a grant from the National Cancer Center and Research Development Fund (28-A-24), and a Senior Investigator Award from the Ontario Institute for Cancer Research to Geoffrey T. Fong.

Conflicts of Interest: GTF and JFT have served as expert witnesses on behalf of governments in litigation involving the tobacco industry. MLG has received a research grant from Pfizer and served as a member of scientific advisory board to Johnson and Johnson. All other authors have no conflicts of interest to declare.

\section{References}

1. World Health Organization. Tobacco: Deadly in Any Form or Disguise. World No Tobacco Day. 2006. Available online: https://www.who.int/tobacco/communications/events/wntd/2006/Report_v8_4May06.pdf (accessed on 5 March 2020).

2. National Center for Chronic Disease Prevention and Health Promotion (US) Office on Smoking and Health. The Health Consequences of Smoking—50 Years of Progress: A Report of the Surgeon General; Centers for Disease Control and Prevention (US): Atlanta, GA, USA, 2014. 
3. American Lung Association. There Are 4000 Chemicals in Every Cigarette. 2016. Available online: https://www.lung.ca/lung-health/smoking-and-tobacco/whats-cigarettes/there-are-4000-chemicals-everycigarette (accessed on 5 March 2020).

4. Czoli, C.D.; Fong, G.T.; Goniewicz, M.L.; Hammond, D. Biomarkers of Exposure among “Dual Users" of Tobacco Cigarettes and Electronic Cigarettes in Canada. Nicotine Tob. Res. 2019, 21, 1259-1266. [CrossRef] [PubMed]

5. Goniewicz, M.L.; Smith, D.M.; Edwards, K.C.; Blount, B.C.; Caldwell, K.L.; Feng, J.; Wang, L.; Christensen, C.; Ambrose, B.; Borek, N.; et al. Comparison of Nicotine and Toxicant Exposure in Users of Electronic Cigarettes and Combustible Cigarettes. JAMA Netw. Open 2018, 1, e185937. [CrossRef] [PubMed]

6. National Academies of Sciences, Engineering, and Medicine. Public Health Consequences of E-Cigarettes; The National Academies Press: Washington, DC, USA, 2018. Available online: https://www.nap.edu/catalog/ 24952/public-health-consequences-of-e-cigarettes (accessed on 5 March 2020).

7. McNeill, A.; Brose, L.S.; Calder, R.; Bauld, L.; Robson, D. Evidence Review of E-Cigarettes and Heated Tobacco Products 2018; A report commissioned by Public Health England; Public Health England: London, UK, 2018. Available online: https://assets.publishing.service.gov.uk/government/uploads/system/uploads/attachment_ data/file/684963/Evidence_review_of_e-cigarettes_and_heated_tobacco_products_2018.pdf (accessed on 5 March 2020).

8. Ramstrom, L.; Borland, R.; Wikmans, T. Patterns of Smoking and Snus Use in Sweden: Implications for Public Health. Int. J. Environ. Res. Public Health 2016, 13, 1110. [CrossRef] [PubMed]

9. Levy, D.T.; Borland, R.; Lindblom, E.N.; Goniewicz, M.L.; Meza, R.; Holford, T.R.; Yuan, Z.; Luo, Y.; O'Connor, R.J.; Niaura, R.; et al. Potential deaths averted in USA by replacing cigarettes with e-cigarettes. Tob. Control. 2018, 27, 18-25. [CrossRef]

10. Leigh, N.J.; Palumbo, M.N.; Marino, A.M.; O'Connor, R.J.; Goniewicz, M.L. Tobacco-specific nitrosamines (TSNA) in heated tobacco product IQOS. Tob. Control. 2018, 27, s37-s38. [CrossRef]

11. Phillip Morris International. IQOS: Tobacco Meets Technology. USA: Phillip Morris International. Available online: https://www.pmi.com/smoke-free-products/iqos-our-tobacco-heating-system (accessed on 5 March 2020).

12. Simonavicius, E.; McNeill, A.; Shahab, L.; Brose, L.S. Heat-not-burn tobacco products: A systematic literature review. Tob. Control. 2019, 28, 582-594. [CrossRef]

13. Mallock, N.; Pieper, E.; Hutzler, C.; Henkler-Stephani, F.; Luch, A. Heated Tobacco Products: A Review of Current Knowledge and Initial Assessments. Front. Public Health 2019, 7, 287. [CrossRef]

14. Centers for Disease Control and Prevention (CDC). Smoking and Tobacco Use. Heated Tobacco Products. Available online: https://www.cdc.gov/tobacco/basic_information/heated-tobacco-products/index.html (accessed on 5 March 2020).

15. Vorster, P.; Mori, M.; Mills, C.; Erskine, A.; Baig, F.; Eggleton, M. Global Tobacco. Next Generation Products: Big in Japan. Global Equity Research Tobacco. Credit Suisse. 2017. Available online: https: //plus.credit-suisse.com/rpc4/ravDocView?docid=_XCsR2AL-YxKG (accessed on 5 March 2020).

16. Philip Morris International. 2019 Fourth-Quarter and Full-Year Results, February 6, 2020. Available online: https://philipmorrisinternational.gcs-web.com/static-files/65f88bac-534b-45f8-ad47-f1f171b0c360 (accessed on 5 March 2020).

17. Dautzenberg, B.; Dautzenberg, M.D. Systematic analysis of the scientific literature on heated tobacco. Rev. Mal. Respir. 2019, 36, 82-103. [CrossRef]

18. World Health Organization. Heated Tobacco Products (HTPs) Information Sheet. WHo/nmH/pnd/17.6 2018. Available online: https:/apps.who.int/iris/bitstream/handle/10665/272875/WHO-NMH-PND-17.6-eng.pdf? ua=1 (accessed on 5 March 2020).

19. Borland, R.; Murray, K.; Gravely, S.; Fong, G.T.; Thompson, M.E.; McNeill, A.; O'Connor, R.J.; Goniewicz, M.L.; Yong, H.H.; Levy, D.T.; et al. A new classification system for describing concurrent use of nicotine vaping products alongside cigarettes (so-called 'dual use'): Findings from the ITC-4 Country Smoking and Vaping wave 1 Survey. Addiction 2019, 114 (Suppl. 1), 24-34. [CrossRef]

20. Kolenikov, S. Calibrating Survey Data using Iterative Proportional Fitting (Raking). Stata J. 2014, 14, $22-59$. Available online: https://journals.sagepub.com/doi/abs/10.1177/1536867X1401400104 (accessed on 5 March 2020). [CrossRef] 
21. Brose, L.S.; Brown, J.; Hitchman, S.C.; McNeill, A. Perceived relative harm of electronic cigarettes over time and impact on subsequent use. A survey with 1-year and 2-year follow-ups. Drug Alcohol Depend. 2015, 157, 106-111. [CrossRef] [PubMed]

22. Farsalinos, K.E.; Romagna, G.; Tsiapras, D.; Kyrzopoulos, S.; Voudris, V. Characteristics, perceived side effects and benefits of electronic cigarette use: A worldwide survey of more than 19,000 consumers. Int. J. Environ. Res. Public Health 2014, 11, 4356-4373. [CrossRef] [PubMed]

23. Persoskie, A.; O’Brien, E.K.; Poonai, K. Perceived Relative Harm of Using E-Cigarettes Predicts Future Product Switching among U.S. Adult Cigarette and E-Cigarette Dual Users. Addiction 2019, 114, 2197-2205. [CrossRef] [PubMed]

24. Tompkins, C.N.E.; Burnley, A.; McNeill, A.; Hitchman, S.C. Factors that influence smokers' and ex-smokers' use of IQOS: A qualitative study of IQOS users and ex-users in the UK. Tob. Control. 2020. Epub ahead of print. [CrossRef]

25. Queloz, S.; Etter, J.F. An online survey of users of tobacco vaporizers, reasons and modes of utilization, perceived advantages and perceived risks. BMC Public Health 2019, 19, 642. [CrossRef]

26. Kim, J.; Yu, H.; Lee, S.; Paek, Y.J. Awareness, experience and prevalence of heated tobacco product, IQOS, among young Korean adults. Tob. Control. 2018, 27, s74-s77. [CrossRef]

27. Kotz, D.; Kastaun, S. E-cigarettes and heat-not-burn products: Representative data on consumer behaviour and associated factors in the German population (the DEBRA study). Bundesgesundheitsblatt Gesundheitsforschung Gesundheitsschutz 2018, 61, 1407-1414. [CrossRef]

28. Institute of Medicine (US) Committee on Preventing Nicotine Addiction in Children and Youths. Growing up Tobacco Free: Preventing Nicotine Addiction in Children and Youths; Lynch, B.S., Bonnie, R.J., Eds.; National Academies Press (US): Washington, DC, USA, 1994.

29. Tabuchi, T.; Shinozaki, T.; Kunugita, N.; Nakamura, M.; Tsuji, I. Study Profile: The Japan “Society and New Tobacco" Internet Survey (JASTIS): A longitudinal internet cohort study of heat-not-burn tobacco products, electronic cigarettes and conventional tobacco products in Japan. J. Epidemiol. 2019, 29, 444-450. [CrossRef]

30. Vorster, P.; Erskine, A.; Baig, F.; Molloy, A. Global Tobacco. Credit Suisse. 2018. Available online: https://plus.credit-suisse.com/rpc4/ravDocView?docid=_XCsR2AL-YxKG (accessed on 5 March 2020).

31. Tabuchi, T.; Gallus, S.; Shinozaki, T.; Nakaya, T.; Kunugita, N.; Colwell, B. Heat-not-burn tobacco product use in Japan: Its prevalence, predictors and perceived symptoms from exposure to secondhand heat-not-burn tobacco aerosol. Tob. Control. 2018, 27, e25-e33. [CrossRef]

32. Sutanto, E.; Miller, C.; Smith, D.M.; O'Connor, R.J.; Quah, A.C.; Cummings, K.M.; Xu, S.; Fong, G.T.; Hyland, A.; Ouimet, J.; et al. Prevalence, Use Behaviors, and Preferences among Users of Heated Tobacco Products: Findings from the 2018 ITC Japan Survey. Int. J. Environ. Res. Public Health 2019, 16, 4630. [CrossRef]

33. Xu, S.S.; Meng, G.; Gravely, S.; Quah, A.C.K.; Ouimet, J.; O'Connor, R.; Yoshimi, I.; Mochizuki-Kobayashi, Y.; Fong, G.T. Reasons for using heated tobacco products among adult current and former smokers in Japan. In Findings from 2018 ITC Japan Survey; Society for Research on Nicotine and Tobacco Annual Meeting: San Francisco, CA, USA, 2019.

34. Bjartveit, K.; Tverdal, A. Health consequences of smoking 1-4 cigarettes per day. Tob. Control. 2005, 14, 315-320. [CrossRef] [PubMed]

35. Tverdal, A.; Bjartveit, K. Health consequences of reduced daily cigarette consumption. Tob. Control. 2006, 15, 472-480. [CrossRef] [PubMed]

36. Popova, L.; Lempert, L.K.; Glantz, S.A. Light and mild redux: Heated tobacco products' reduced exposure claims are likely to be misunderstood as reduced risk claims. Tob. Control. 2018, 27, s87-s95. [CrossRef] [PubMed]

(C) 2020 by the authors. Licensee MDPI, Basel, Switzerland. This article is an open access article distributed under the terms and conditions of the Creative Commons Attribution (CC BY) license (http://creativecommons.org/licenses/by/4.0/). 